\title{
Radionuclide assessment of left ventricular function in hypertrophic cardiomyopathy
}

\author{
D.D. Sugrue and W.J. McKenna ${ }^{1}$
}

Division of Cardiovascular Disease, Mayo Clinic, Rochester, Minnesota 55905, U.S.A. and ${ }^{1}$ Division of

Cardiovascular Disease, Royal Postgraduate Medical School, DuCane Road, London W12 0HS, UK.

\begin{abstract}
Summary: To determine the relationship of left ventricular function and ventricular tachycardia, 48 hour ECG monitoring and technetium-99m gated equilibrium radionuclide angiography were performed in 84 consecutive patients with hypertrophic cardiomyopathy and sinus rhythm. Measurements of ejection fraction (EF), peak ejection rate (PER, edv/s), peak filling rate (PFR, edv/s) and time to peak filling rate (PFR, ms) were derived from radionuclide activity time curves generated from data acquired in list-mode. Left ventricular function was compared in patients with and without ventricular tachycardia. Left ventricular ejection fraction was significantly lower in 16 patients with ventricular tachycardia compared to 68 patients without $(67 \pm 17$ vs $78 \pm 10, P<0.05)$ and time to peak filling rate was significantly prolonged (152 \pm 32 vs $120 \pm 36, P<0.05)$. Thus patients with hypertrophic cardiomyopathy at greatest risk of sudden death had significant impairment of systolic and diastolic left ventricular function.
\end{abstract}

\section{Introduction}

Abnormalities of systolic and diastolic function are common in hypertrophic cardiomyopathy. Standard methods for assessing these abnormalities by invasive techniques or using echocardiography have several potential limitations; the technology is either complex and not widely available or measurements are not very reproducible. For example, construction of pressure volume loops requires cardiac catheterization and data interpretation may be difficult; digitized echocardiography is not widely available and measurements made using this technique are critically dependent on ventricular geometry.

High temporal resolution gated radionuclide cine angiography offers the potential for serial non-invasive assessment of left ventricular ejection and filling; the technical success rate of the technique is high, error margins for measurements obtained using the technique are acceptably low (Sugrue et al., 1985) and radionuclide measurements correlate reasonably with similar measurements obtained using contrast angiography (Sugrue et al., 1984a). We have used this technique in patients with hypertrophic cardiomyopathy in order to assess the relationship between left ventricular gradients and stroke volume ejected during early and late systole (Sugrue et al., 1984b), and to study the effect of an antiarrhythmic drug (amiodarone) on left ventricular ejection and

Correspondence: W.J. McKenna, M.D. filling (Sugrue et al., 1984c). Recently we have used a radionuclide technique to study the relationship of clinical and prognostic features to left ventricular function in hypertrophic cardiomyopathy.

\section{Materials and methods}

Radionuclide studies were performed at rest in the supine position using a large field of view rotating gamma camera. Red blood cells were labelled in vivo using $15 \mathrm{mCi}$ of technetium-99m and images were acquired in list-mode. Data were acquired from 600 to 900 consecutive cycles and stored on computer for subsequent analysis. An R-R interval histogram was constructed and cycles more than $20 \%$ from the mean cycle length were rejected. A background corrected composite left ventricular activity time curve was generated at a frame rate of $10-25 \mathrm{~ms}$ per frame by combined forward and reverse gating from the $\mathbf{R}$ wave. A standard count-based method was used for calculation of left ventricular ejection fraction (LVEF) (Sugrue et al., 1985). Peak rates of ejection and filling were computed automatically in left ventricular counts per second, were normalized to end-diastolic counts and expressed as change from end-diastolic volume per second (edv/s). This approach does not assume knowledge of absolute left ventricular volume. Time to end systole (TES), in milliseconds, was

(C) The Fellowship of Postgraduate Medicine, 1986 
defined as the interval from the $\mathrm{R}$-wave to the minimum of the curve; time to peak ejection rate (TPER) was defined as the interval from the $R$-wave to the point of peak ejection and time to peak filling rate (TPFR) was defined as the interval from the minimum of the curve to the point of peak filling (Figure 1).

Ambulatory electrocardiographic monitoring was performed for at least 48 hours in each patient off all antiarrhythmic medications.

Eighty-four consecutive patients with hypertrophic cardiomyopathy were studied. They were aged 7-72 years, mean $44 ; 46$ were male and 38 female. Values for radionuclide indices of left ventricular ejection and filling were determined in 28 normal volunteers. (The values given are mean \pm s.d.)

\section{Results}

Forty-five of 84 patients (54\%) had an ejection fraction above the upper limit of normal $(>75 \%), 27$ of $84(32 \%)$ had above normal peak ejection rates $(>4.09 \mathrm{edv} / \mathrm{s})$ and 24 of $84(29 \%)$ had a shortened $(<144 \mathrm{~ms})$ time to peak ejection rate. Five of 84 patients $(6 \%)$ had an ejection fraction below the lower limits of normal $(<53 \%), 7$ of $84(8 \%)$ had decreased peak ejection rate $(>2.64 \mathrm{edv} / \mathrm{s})$ and 3 of $84(4 \%)$ had a prolonged time to peak ejection rate $(>264 \mathrm{~ms})$.

Twenty-one patients $(25 \%)$ had supranormal $(>3.89 \mathrm{edv} / \mathrm{s})$ peak filling rates and $4(5 \%)$ had shortened time to peak filling rates $(<61 \mathrm{~ms})$. Fifteen of $84(18 \%)$ patients had below normal peak filling rates $(<2.40 \mathrm{edv} / \mathrm{s})$ while $25(30 \%)$ had prolonged $(>191 \mathrm{~ms})$ time to peak filling rate.

Sixteen patients $(19 \%)$ had one or more episodes of ventricular tachycardia $>3$ consecutive beats) documented during 48 hour ambulatory monitoring. In those patients with ventricular tachycardia, mean

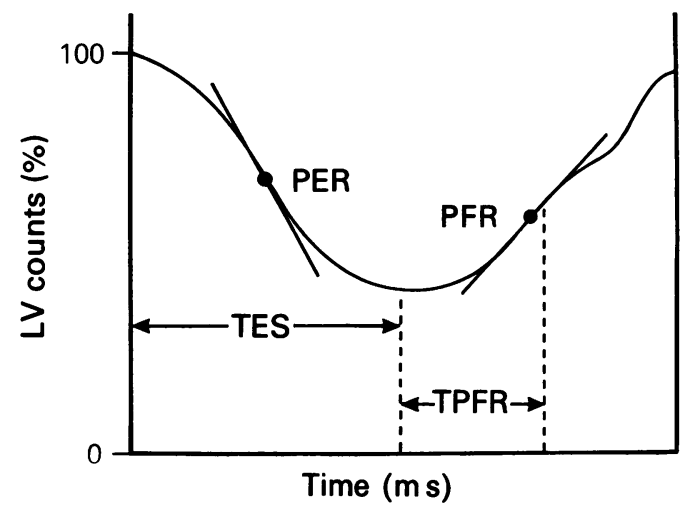

Figure 1 A schematic representation of a radionuclide activity-time curve. Ejection and filling rates were normalized to end-diastolic counts and expressed as change from end-diastolic volume per second (edv/s). Time to peak ejection rate (TPER) was defined as the interval from the beginning of the curve to the point of peak ejection and time to peak filling rate (TPFR) as the interval from end-systole (minimum of the curve) to the point of peak filling. PER = peak ejection rate; $P F R=-$ peak filling rate; TES = time to end-systole.

left ventricular ejection fraction was decreased ( $66 \pm 17$ vs $78 \pm 10 \%, P<0.05)$ and time to peaks filling rate was prolonged $(152 \pm 37 v s 120 \pm 36 \mathrm{~ms}$ $P<0.05)$ (Table I).

\section{Discussion}

These results emphasize the heterogeneity of left ventricular dysfunction in patients with hypertrophic cardiomyopathy. This spectrum of abnormalities in diastolic function has been previously noted using a

Table I Relationship of radionuclide measurements of left ventricular ejection and filling to previously documented ventricular tachycardia in 84 patients with hypertrophic cardiomyopathy

\begin{tabular}{|c|c|c|c|}
\hline & $\begin{array}{c}\text { Ventricular } \\
\text { tachycardia } \\
\mathrm{n}=16(19 \%)\end{array}$ & $\begin{array}{c}\text { No ventricular } \\
\text { tachycardia } \\
\mathrm{n}=68(81 \%)\end{array}$ & \\
\hline Ejection fraction (\%) & $67 \pm 17$ & $78 \pm 10$ & $P<0.05$ \\
\hline Peak ejection rate (edv/s) & $3.3 \pm 1.0$ & $3.9 \pm 1.0$ & NS \\
\hline Time to peak ejection rate (ms) & $174 \pm 35$ & $174 \pm 34$ & NS \\
\hline Time to end-systole (ms) & $375 \pm 49$ & $358 \pm 53$ & NS \\
\hline Peak filling rate (edv/s) & $2.8 \pm 1.2$ & $3.3 \pm 1.2$ & NS \\
\hline Time to peak filling rate (ms) & $152 \pm 37$ & $120 \pm 36$ & $P<0.05$ \\
\hline Heart rate (beats/min) & $64 \pm 9$ & \pm 8 & NS \\
\hline Systolic blood pressure (mm Hg) & $127 \pm 11$ & $131 \pm 12$ & NS \\
\hline Diastolic blood pressure $(\mathrm{mm} \mathrm{Hg})$ & $78 \pm 9$ & $75 \pm 11$ & NS \\
\hline
\end{tabular}

NS = not significant 
variety of techniques for assessing $\mathrm{LV}$ filling, including echocardiography (St. John Sutton et al., 1978), contrast angiography (Sanderson et al., 1977) and radionuclide angiography (Bonow et al., 1981). Impairment of systolic function in hypertrophic cardiomyopathy has been less well documented although Bonow et al. (1981) noted a subnormal left ventricular ejection fraction in one of 40 patients studied, using a radionuclide technique similar to ours. It has been suggested that, as the disease progresses, the left ventricle becomes dilated with loss of outflow tract gradient and by implication, a decline of systolic pump function (Goodwin \& Oakley, 1972).

The relationship between impairment of systolic and diastolic function and previously documented ventricular tachycardia is of some interest. Because of the cross-sectional nature of the study, the results do not provide direct evidence that impairment of filling per se is an independent predictor of subsequent sudden death. However, we have previously performed a prospective comparison of contrast angiographic measurements of ejection and filling in 88 patients with hypertrophic cardiomyopathy of whom 11 subsequently died during a mean follow-up period of 8 years, to similar measurements in survivors (Newman

\section{References}

BONOW, R.O., ROSING, D.R., BACHARACH, S.L., GREEN, M.V., KENT, K.M., LIPSON, L.C., MARON, B.J., LEON, M.B. \& EPSTEIN, S.E. (1981). Effects of verapamil on left ventricular systolic function and diastolic filling in patients with hypertrophic cardiomyopathy. Circulation, 64, 787.

GOODWIN, J.F. \& KRIKLER, D.M. (1976). Arrhythmia as a cause of sudden death in hypertrophic cardiomyopathy. Lancet, ii, 937.

GOODWIN, J.F. \& OAKLEY, C.M. (1972). The cardiomyopathies. British Heart Journal, 34, 542.

NEWMAN, H., SUGRUE, D., OAKLEY, C.M., GOODWIN, J.F. \& McKENNA, W.J. (1985). Relation of left ventricular function and prognosis in hypertrophic cardiomyopathy: an angiographic study. Journal of the American College of Cardiology, 5, 1064.

ST. JOHN SUTTON, M.G., TAJIK, A.J., GIBSON, D.G., BROWN, D.J., SEWARD, J.B. \& GIULIANI, E.R. (1978). Echocardiographic assessment of left ventricular filling and septal and posterior wall dynamics in idiopathic hypertrophic subaortic stenosis. Circulation, 57, 512.

SANDERSON, J.E., GIBSON, D.G., BROWN, D.J. \& GOODWIN, J.F. (1977). Left ventricular filling in hypertrophic cardiomyopathy. An angiographic study. British Heart Journal, 39, 661 . et al., 1985). Patients who died suddenly had a significantly lower normalized peak rate of left ventricular ejection and filling at diagnosis compared to survivors. The results of these two studies are concordant and it therefore seems likely that in adult patients, impairment of left ventricular function predicts poor prognosis. The relative power and independence of these individual predictors of sudden death remains to be determined. Although ventricular tachycardia is the presumed antecedent of sudden death in most, though perhaps not all patients with this disease, it has been suggested that a primary haemodynamic mechanism, i.e. rapid heart rates, coupled with impairment of left ventricular filling may lead to an acute decrease in stroke output with a concomitant decline in diastolic coronary flow and cardiovascular collapse (Goodwin \& Krikler, 1976). The results of our studies support this hypothesis and suggest that impairment of left ventricular filling may be an essential substrate which determines whether or not ventricular tachycardia is well tolerated or precipitates ventricular fibrillation. It remains to be seen if drugs such as calcium antagonists, which improve diastolic filling, also improve prognosis.

SUGRUE, D.D., DICKIE, S., NEWMAN, H., MYERS, M.J., LAVENDER, J.P. \& MCKENNA, W.J. (1984a). Comparison of equilibrium radionuclide and contrast angiographic measurements of left ventricular peak ejection and filling rates and their time intervals. Nuclear Medicine Communications, 5, 619.

SUGRUE, D.D., MCKENNA, W.J., DICKIE, S., MYERS, M.J., LAVENDER, J.P., OAKLEY, C.M. \& GOODWIN, J.F. (1984b). Relation between left ventricular gradient and relative stroke volumes ejected in early and late systole in hypertrophic cardiomyopathy. British Heart Journal, 52, 602.

SUGRUE, D.D., DICKIE, S.S., MYERS, M.J., LAVENDER, J.P. \& MCKENNA, W.J. (1984c). Effect of amiodarone on left ventricular ejection and filling in hypertrophic cardiomyopathy as assessed by radionuclide angiography. American Journal of Cardiology, 54, 1054.

SUGRUE, D.D., DICKIE, S., MYERS, M.H., LAVENDER, J.P. \& McKENNA, W.J. (1985). Errors in gated equilibrium radionuclide measurements of resting left ventricular ejection and filling. European Heart Journal (in press). 